\title{
Llanto excesivo en los primeros meses de vida. Revisión
}

\author{
Excessive crying in infants in the first months of life. Review
}

Prof. Dr. Patricio Kenny ${ }^{a}$

\begin{abstract}
RESUMEN
El comportamiento caracterizado por llanto e irritabilidad, una de las presentaciones clínicas más frecuentes en los primeros meses de vida, surge de una compleja interacción de factores culturales, psicosociales, ambientales y biológicos. El 5\% de los casos presenta alguna afección orgánica. Un enfoque holístico de tipo biopsicosocial del problema parece ser el más conveniente.

El presente manuscrito examina la evidencia reciente con el propósito de actualizar el manejo clínico de esta afección e intentar redefinirla.

Palabras clave: lactancia materna, cólico, lactante, llanto, genio irritable, sueño.
\end{abstract}

\begin{abstract}
Cry-fuss problems are among the most common clinical presentations in the first few months of life. Cry-fuss behaviour emerges out of a complex interplay of cultural, psychosocial, environmental and biologic factors, with organic disturbance implicated in only $5 \%$ of cases. A holistic biopsychosocial approach can be most effective. This article reviews recent evidence to update clinical management, and will attempt to reconceptualize infant colic definition.

Key words: breast-feeding, colic, infant, crying, irritable mood, sleep.
\end{abstract}

http:/ /dx.doi.org/10.5546/aap.2016.368

a. Facultad de Ciencias Médicas.

Pontificia Universidad Católica Argentina.

Correspondencia: Dr. Patricio Kenny, patriciokenny@uca.edu.ar

Financiamiento:

Ninguno.

Conflicto de intereses: Ninguno que declarar.

Recibido: 8-10-2015. Aceptado: 22-2-2016

\section{INTRODUCCIÓN}

El lactante con llanto e irritabilidad en los primeros tres a cuatro meses de vida representa una de las consultas más frecuentes en el consultorio de un pediatra general.

Si bien el problema suele ser benigno y autolimitado en el tiempo, no se trata de una afección trivial, ya que, ocasionalmente, puede anunciar algo más serio y de largo alcance. ${ }^{1}$

Estudios realizados en cohortes comunitarias señalan que uno de cada cinco padres afirma que su bebé, si bien se encuentra saludable, tiene problemas de llanto e irritabilidad a los dos meses de edad..$^{2,3}$ Este comportamiento emerge de una compleja interacción de factores culturales, psicosociales, ambientales y biológicos, donde la enfermedad orgánica representa un $5 \%$ de los casos en atención primaria y un $10 \%$ de aquellos derivados a un especialista. ${ }^{4,5}$

La presente es una revisión de la evidencia que pretende ofrecer al pediatra general una guía actualizada para el manejo de los niños con llanto excesivo en los primeros meses de vida.

\section{Llanto normal y llanto} problemático. Definiciones

"Cólico" es un término ambiguo y desactualizado. Usualmente, se lo describe como un "comportamiento caracterizado por un llanto paroxístico y excesivo". El término "cólico" deriva de la palabra griega kolikos o kolon, que sugiere que el origen del problema se encuentra en el tracto digestivo. Es más probable que ocurra por las tardes y sin una causa identificable. Los episodios de "cólicos" acontecen entre las 2 semanas y los 4 meses de vida, y son difíciles de consolar. Se trata de un problema aún no bien definido y difícil de abordar desde el punto de vista diagnóstico y terapéutico. Esto se debe, en parte, a que el llanto suele ser el único síntoma de presentación, y este como tal no puede ser menos específico. Representa uno de los motivos de consulta y desafíos más frecuentes que le llegan al pediatra en los primeros 3 meses de vida del bebé.

La definición más empleada de "cólico" es la acuñada por Wessel y cols., ${ }^{6}$ y se sustenta en la cuantificación del llanto (paroxismos de llanto que duran más de 3 horas por día, más de 3 días por semana en cualquier semana y, por lo menos, durante 3 
semanas). Sin embargo, tal vez, la mejor definición del problema surja de la misma percepción de los padres, referida como "cualquier comportamiento de llanto informado como problemático en los primeros 3-4 meses de vida".

Aun cuando las definiciones del "lactante que llora" varían considerablemente, por razones prácticas, se emplearán los términos "llanto e irritabilidad", "llanto excesivo", "cólicos funcionales" y "el bebé intranquilo o inquieto" en forma intercambiable para hacer referencia a cualquier comportamiento de llanto que los padres informan como problemático en los primeros 3-4 meses de vida.

\section{¿Puede el llanto problemático en lactantes tener consecuencias desfavorables?}

La mayoría de los bebés tiene episodios de llanto inconsolable en los primeros meses de vida; sin embargo, el llanto problemático suele ser más frecuente y de mayor duración. ${ }^{1}$

Según estudios longitudinales, el 5\% de los bebés que lloran continúan con este comportamiento a los 5 meses de edad, ${ }^{2,7}$ empero, no es posible predecir cuál de ellos continuará llorando persistentemente más allá de los 3-4 meses.

Si bien la mayoría de los bebés que lloran de manera excesiva en los primeros 3 meses no exhiben efectos adversos en el largo plazo, el llanto excesivo no es un problema trivial, debido a que expone a las familias a un elevado y mensurable nivel de estrés.

En un estudio realizado sobre 3259 familias, $6 \%$ de los padres afirmaron retrospectivamente haber cometido abuso físico de sus hijos durante alguna crisis de llanto. ${ }^{8}$

Un estudio multicéntrico encontró que las madres de bebés con cólicos o llanto excesivo tenían puntajes más altos en la escala de depresión posnatal de Edimburgo, con mayores probabilidades de puntajes elevados a los 6 meses, aún cuando el llanto del bebé hubiera mermado. ${ }^{9}$ Esto sustenta los hallazgos de investigaciones previas que vinculan el llanto e irritabilidad con ansiedad y depresión materna perinatal. ${ }^{1}$

Otro estudio prospectivo realizado en

Figura 1. Abordaje del llanto excesivo en los primeros meses de vida

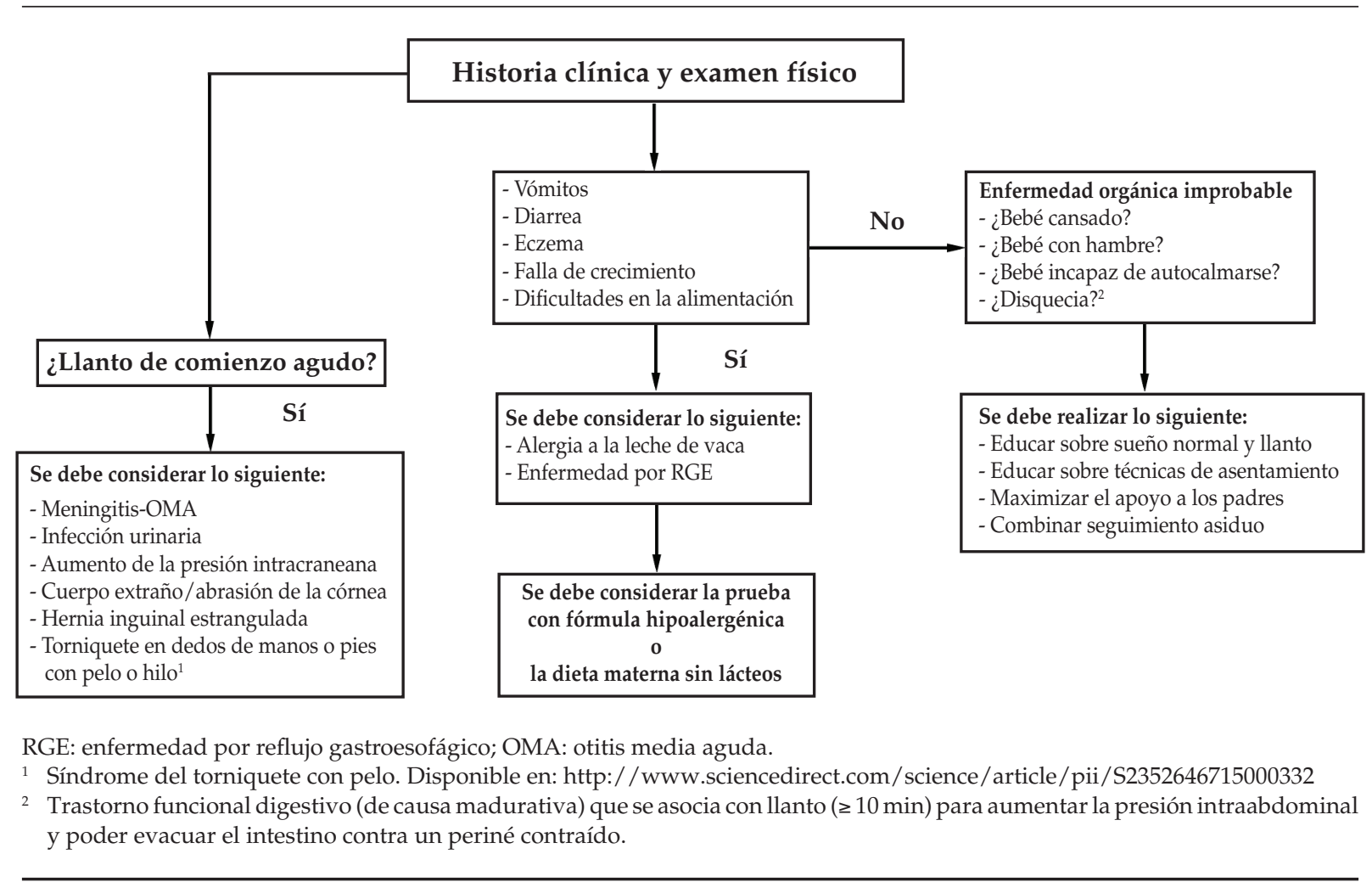


700 pares de madres e hijos encontró que el diagnóstico de cólicos se asoció con una lactancia natural acortada. ${ }^{10}$

Un metaanálisis que recopila 22 estudios longitudinales encontró que los bebés con problemas de llanto y/o irritabilidad que persistieron hasta los 5 meses de edad presentaban un riesgo elevado de padecer problemas de conducta durante la niñez. ${ }^{11}$

El riesgo parece ser mayor en aquellos lactantes que también presentan problemas relacionados con el sueño y la alimentación (o ambos) a los 5 meses y en familias que enfrentan situaciones adversas o tienen determinados factores de riesgo psicosocial. ${ }^{12}$

\section{¿Qué problemas tratables pueden existir detrás del llanto e irritabilidad problemáticos?}

Cuando los padres se presentan a la consulta con un bebé que llora mucho, deberán descartarse afecciones médicas serias que pueden ocultarse detrás de dichos síntomas.

\section{Reflujo gastroesofágico}

Si bien dos consensos internacionales ${ }^{13,14}$ integrados por comités de expertos coinciden en que la enfermedad por reflujo gastroesofágico (ERGE) ácido péptica no es causa de llanto e irritabilidad en los primeros meses de vida, muchos padres (y también pediatras) con bebés que lloran siguen considerando que los problemas de llanto e irritabilidad son causados por "reflujo", o bien que el "cólico" siempre es causado por un dolor de origen digestivo. ${ }^{15,16} \mathrm{~A}$ pesar de que existe una asociación entre erosiones en la mucosa esofágica, cambios microscópicos de la ERGE, y la exposición ácida prolongada en el esófago inferior, evidencia creciente señala que el reflujo ácido no es una explicación plausible del llanto en lactantes con ERGE sintomática. ${ }^{17}$

Si el dolor provocado por el reflujo ácido que interactúa a nivel celular con nociceptores en el esófago ("ardor retroesternal") fuera la causa del llanto en bebés con ERGE, la poderosa supresión ácida gástrica de los inhibidores de la bomba de protones (IBP) debería reducir o controlar el llanto. Sin embargo, esto no ocurre. ${ }^{17,18}$ Además, el uso indiscriminado de los IBP, también conocidos como "prazoles", puede incrementar el riesgo de neumonía, gastroenteritis y otras infecciones, así como malabsorción de nutrientes, que incluyen magnesio, calcio, $\mathrm{y}$ vitamina $\mathrm{B}_{12}$, $\mathrm{y}$ alteraciones en la microbiota intestinal. ${ }^{19}$

Estas drogas no están indicadas para un bebé cuyo único problema es el llanto excesivo, incluso cuando el llanto se asocia con arqueo hacia atrás y rechazo del alimento. ${ }^{20,21}$ Por lo tanto, se puede tranquilizar a los padres al explicarles que, debido al efecto "buffer" (del inglés, "neutralizante") de la leche, el material que refluye tiene una acidez neutra durante dos horas después de cada comida y que, por ello, no es causa de esofagitis ni malestar en el bebé de esta edad. ${ }^{22}$

\section{Problemas con la alimentación y durante ella}

Existe un vínculo importante entre las dificultades alimentarias y el llanto excesivo. Un manejo inadecuado de la lactancia materna genera una confusión importante en la investigación de los bebés que lloran. ${ }^{23}$ Estudios observacionales han demostrado una asociación entre factores de riesgo psicosociales y biológicos en pares de madres e hijos y una afectación en la mutua regulación de la alimentación, que resulta en un afianzamiento de aquellos patrones que derivan en una alimentación problemática. ${ }^{24}$ Las dificultades en la lactancia materna -tales como problemas en el acople y posicionamiento entre madre e hijo, sobrecarga funcional de lactosa, disfunción motora oral, anquiloglosia, y problemas en el procesamiento sensorialpodrían colocar a los bebés susceptibles en riesgo de afianzar conductas de aversión hacia los alimentos además del llanto y la irritabilidad. . $^{2,26}$

Muchos clínicos pediatras, enfermeras pediátricas y neonatales, puericultoras y gastroenterólogos infantiles en todo el mundo, que incluyen a aquellos con actitudes positivas hacia la lactancia materna, no han advertido su falta de conocimientos en relación con la identificación y el manejo de los problemas en la alimentación. . $^{27,28}$

\section{Sobrecarga funcional de lactosa}

En la medida que una mamada progresa y el volumen de leche consumido por el bebé se reduce, la proporción de lípidos en esa comida se incrementa. Esta leche más cremosa enlentece el tiempo de tránsito intestinal del bebé y estimula la hormona colecistoquinina para provocar saciedad. La sobrecarga funcional de lactosa ocurre cuando las mamadas no contienen suficiente grasa, lo que resulta en un tránsito intestinal rápido.

La lactosa no digerida -debido al escaso tiempo de exposición a la lactasa del ribete en cepillo- es fermentada en el colon, y el bebé puede no sentirse saciado, tener deposiciones 
semilíquidas, explosivas y grumosas, un abdomen timpánico, llanto y el deseo de ser alimentado muy seguido. ${ }^{29}$

Un estudio controlado y aleatorizado sobre 77 lactantes de 5 semanas de vida mostró que aquellos con cólicos tenían concentraciones de la hormona colecistoquinina en plasma más baja. ${ }^{30}$

Un ensayo clínico aleatorizado de 302 pares de madres e hijos, que comparó diferentes técnicas de amamantamiento, encontró también que la sobrecarga funcional de lactosa estaba significativamente asociada con el llanto de los bebés. ${ }^{31}$

\section{Alergia alimentaria}

La alergia a las proteínas de la leche de vaca (APLV) es la alergia alimentaria más frecuente en la edad pediátrica y parece ser uno de los principales contribuyentes al llanto e irritabilidad en los primeros meses de vida. Afecta, por lo menos, a un $2 \%$ de los lactantes. ${ }^{32,33}$

Lothe y Lindberg ${ }^{34}$, y luego Iacono ${ }^{35} \mathrm{y}$ Hill, ${ }^{36}$ fueron los primeros en demostrar una alta prevalencia de "cólicos" en lactantes con APLV. Poco tiempo después, analizando una cohorte secuencial de 100 pacientes con APLV documentada por la prueba de desafío, Hill y cols. observaron que el $44 \%$ de los lactantes estudiados mostraron un comportamiento irritable y "colicoso" durante el desafío con leche de vaca. ${ }^{37}$ Desde entonces, múltiples ensayos clínicos han demostrado un beneficio en el tratamiento de los lactantes con cólicos, tanto con soja como con hidrolizados extensos de proteínas, incluso cuando ningún otro síntoma era evidente. ${ }^{38,39}$

En la actualidad, no hay evidencia convincente de que otras alergias alimentarias fuera de la alergia a la leche de vaca puedan provocar llanto e irritabilidad en los primeros meses de vida. Sin embargo, un ensayo clínico controlado y aleatorizado de 107 lactantes con cólicos concluyó que una dieta materna con bajo contenido de alérgenos alimentarios -lácteos, soja, trigo, frutos secos y pescado- acortó la duración del llanto en los bebés investigados. ${ }^{40}$

Existe, además, un subgrupo de lactantes con alergia alimentaria susceptible a múltiples proteínas de la dieta, una forma rara de alergia alimentaria no mediada por IgE, que se manifiesta con llanto grave y persistente. ${ }^{41}$ Estos bebés desarrollan irritabilidad grave luego de la ingesta de lecha materna o fórmula a base de leche de vaca, soja, o bien un hidrolizado extenso de proteínas. ${ }^{38} \mathrm{La}$ ausencia de eczema en la mayoría de estos lactantes con llanto persistente sugiere un mecanismo no mediado por IgE. Del mismo modo, la asociación de "alergia a múltiples proteínas de la dieta" con cólicos graves condujo a la hipótesis de que el llanto persistente en los primeros meses de la vida podría ser una manifestación de alergia alimentaria no mediada por $\operatorname{IgE} .^{35}$

Las dietas de eliminación en la madre que amamanta a su hijo reducen la secreción de proteínas alimentarias a través de la leche materna y podrían ofrecer un tratamiento efectivo en una proporción de bebés amamantados con cólicos. Jakobsson y Lindberg ${ }^{42}$ observaron que un tercio de los lactantes con cólicos alimentados exclusivamente con lactancia materna mejoraron luego de la eliminación de la leche de vaca y empeoraron con su reintroducción. Evans y cols.,${ }^{43}$ sin embargo, no confirmaron estos hallazgos.

Un ensayo clínico más reciente de Hill y cols. ${ }^{40}$ examinó el rol de una dieta de exclusión hipoalergénica amplia en 91 lactantes con cólicos, con lactancia materna exclusiva y con menos de 6 semanas de vida.

Las madres fueron asignadas, en forma aleatoria, a una dieta baja en alérgenos (que evitaba la leche de vaca, soja, trigo, huevo, maní, nueces, pescado y mariscos) o a una dieta control irrestricta. Significativamente, más lactantes respondieron a la dieta baja en alérgenos, con una reducción de la duración del llanto e irritabilidad por $48 \mathrm{~h}$ sustancialmente menor $(74 \%$ vs. $37 \%$; RRa: $37 \%$ ) cuando se los comparó con aquellos lactantes cuyas madres recibieron una dieta control.

Varios ensayos clínicos, así como un metaanálisis, han evaluado los efectos de las fórmulas hipoalergénicas en cólicos y han demostrado un beneficio significativo y apreciable. ${ }^{42,44}$

La falta de respuesta al tratamiento con fórmulas hipoalergénicas puede, en algunos lactantes, deberse a una "alergenicidad residual" presente en las fórmulas extensamente hidrolizadas, derivadas tanto de caseína como de las proteínas del suero de la leche. ${ }^{38,45,46}$ En aquellos lactantes que son intolerantes a hidrolizados extensos, existe la opción de otras fórmulas aun más hidrolizadas que reemplazan completamente la proteína intacta por aminoácidos libres. Estas fórmulas han demostrado ser efectivas y seguras. ${ }^{47,48}$ 


\section{Infección}

En un lactante intranquilo e irritable, debe descartarse infección. La meningitis y la otitis media aguda representan, por sus implicancias, los primeros diagnósticos para descartar.

En un estudio retrospectivo de 237 lactantes de menos de un año de vida que se presentaron en una sala de emergencias debido a llanto inconsolable, se encontró que un 5\% de ellos tenía infección urinaria. ${ }^{49}$ Sin embargo, en un bebé afebril con menos de 3 meses de vida, en ausencia de signos y síntomas de enfermedad, el llanto excesivo no requiere un análisis de orina inmediato. ${ }^{50}$

\section{Intolerancia transitoria a la lactosa (insuficiencia ontogénica de lactasa)}

La intolerancia transitoria a la lactosa ha sido identificada como posible causa de cólicos en los primeros meses de vida. En 1998 y 2001, Kearney y cols. y Kanabar y cols., respectivamente, demostraron que el llanto problemático de los primeros meses de vida podía deberse, en un significativo número de los casos, a una deficiencia de lactasa relativa y transitoria, que podía reflejar la inmadurez del tracto digestivo. La administración de lactasa, ya sea en bebés alimentados con leche materna o fórmula, mostró una reducción marcada y estadísticamente significativa en la excreción de hidrógeno en la prueba del aire espirado. Del mismo modo, se observó una marcada reducción en la duración del llanto en aquellos bebés que cumplieron con el protocolo. ${ }^{51,52}$

\section{Migraña}

Un reciente metaanálisis reveló que el cólico podría tratarse de una forma de cefalea migrañosa, más que, como se ha propuesto desde un principio, una afección proveniente del aparato digestivo. El análisis incluyó 3 estudios con un total de 891 pacientes. Uno de esos estudios indicó que existía mayor probabilidad de cólicos en lactantes cuyas madres padecían cefalea migrañosa. Los otros dos indicaron que los lactantes con cólicos eran más propensos a experimentar migraña en la niñez y la adolescencia. Empleando, en su análisis, un modelo de efectos aleatorios agrupados, Gelfand y cols. encontraron que el OR (del inglés, odds ratio; significa "índice de probabilidades") para una asociación entre migraña y cólico alcanzó el 5,6. ${ }^{53,54}$ En un análisis secundario, que incluyó los dos estudios adicionales, el OR fue de 3,2. ${ }^{53} \mathrm{En}$ un estudio reciente proveniente de un grupo de investigadores finlandeses, el RR (risk ratio) de migraña en niños con cólicos fue de $2,8 .{ }^{55}$

\section{Otras opciones terapéuticas}

Diversas investigaciones han analizado otras opciones terapéuticas farmacológicas y no farmacológicas para tratar el llanto excesivo del lactante. Una breve síntesis se encuentra como anexo electrónico.

\section{CONCLUSIÓN}

Es necesario redefinir el término "cólico", evitando asignarle cualquier connotación etiológica. La nueva definición debería ser aquella que mejor se ajustara a la realidad objetiva que perciben padres y cuidadores, y que promoviera una respuesta rápida y operativa por parte de los profesionales de la salud.

Una opción podría ser la siguiente: "llanto problemático", "cualquier comportamiento de llanto que es informado por los padres como problemático en los primeros 3-4 meses de vida".

La Real Academia Española define el término "problemático" como "algo o alguien que presenta dificultades o que causa problemas".

Las dificultades pueden provenir tanto del niño como de sus padres. Los problemas son los que les ocasionan el llanto al mismo bebé y también a sus cuidadores.

La causa de llanto problemático en el lactante durante los primeros meses de vida es multifactorial. Luego de evaluar las dificultades en la alimentación, los problemas de salud mental de la madre luego del parto y haber excluido una eventual enfermedad subyacente, que incluye alergia a la leche de vaca, se recomienda actuar tempranamente con un abordaje individual adaptado y centrado en cada madre y su familia. Un enfoque holístico de tipo biopsicosocial del problema podría ser útil y conveniente. (Figura 1)

La colaboración entre profesionales con experiencia y conocimientos actualizados será necesaria si se pretende ofrecer un abordaje efectivo y evitar complicaciones.

\section{REFERENCIAS}

1. Barr RG, Paterson JA, MacMartin LM, Lehtonen L, Youmg SN. Prolonged and unsoothable crying bouts in infants with and without colic. J Dev Behav Pediatr 2005;26(1):14-23.

2. Wake M, Morton-Allen E, Poulakis Z, Hiscock H, et al. Prevalence, stability, and outcomes of cry-fuss and sleep problems in the first 2 years of life: prospective communitybased study. Pediatrics 2006;117(3):836-42.

3. Wolke D, Samara M, Alvarez M. Meta-analysis of fuss/ cry durations and colic prevalence across countries. En: 1th International Infant Cry Research Workshop: Infant Crying and 
Parenting: Brain, Behavior and Hormones. The Netherlands; 2011 Jun 08-10.

4. Gormally SM, Barr RG. Of clinical pies and clinical clues: proposal for a clinical approach to complaints of early crying and colic. Ambul Child Health 1997;3:137-53.

5. Miller AR, Barr RG. Infantile colic: is it a gut issue? Pediatr Clin North Am 1991;38(6):1407-23.

6. Wessel MA, Cobb JC, Jackson EB, Harris GS Jr, Detwiler AC. Paroxysmal fussing in infancy, sometimes called "colic". Pediatrics 1954;14(5):421-35.

7. Schmid G, Schreier A, Meyer R, Wolke D. A prospective study on the persistence of infant crying, sleeping and feeding problems and preschool behaviour. Acta Paediatr 2010;99(2):286-90.

8. Reijneveld SA, van der Wal M, Brugman E, Sing RA, Verloove-Vanhorick S. Infant crying and abuse. Lancet 2004;364(9442):1340-2.

9. Vik T, Grote V, Escribano J, Socha J, et al. Infantile colic, prolonged crying and maternal postnatal depression. Acta Paediatr 2009;98(8):1344-8.

10. Howard C, Lanphear N, Lanphear B, Eberly S, Lawrence R. Parental responses to infant crying and colic: the effect on breastfeeding duration. Breastfeed Med 2006;1(3):146-55.

11. Hemmi MH, Wolke D, Schneider S. Associations between problems with crying, sleeping and/or feeding in infancy and long-term behavioural outcomes in childhood: a metaanalysis. Arch Dis Child 2011;96(7):622-9.

12. Schmid G, Schreier A, Meyer R, Wolke D. Predictors of crying, feeding and sleeping problems: a prospective study. Child Care Health Dev 2011;37(4):493-502.

13. Sherman P, Hassall E, Fagundes-Neto U, Gold B, et al. A global, evidence-based consensus on the definition of gastroesophageal reflux disease in the pediatric population. Am J Gastroenterol 2009;104(5):1278-95.

14. Vandenplas Y, Rudolph CD, Di Lorenzo C, Hassall E, et al. Pediatric gastroesophageal reflux clinical practice guidelines: Joint recommendations of the North American Society of Pediatric Gastroenterology, Hepatology, and Nutrition and the European Society of Pediatric Gastroenterology, Hepatology, and Nutrition (ESPGHAN). J Pediatr Gastroenterol Nutr 2009;49(4):498-547.

15. Scherer L,Zikmund-Fisher B, Fagerlin A, Tarini B. Influence of "GERD" label on parents' decision to medicate infants. Pediatrics 2013;131(5):839-45.

16. Carey WB. The hazards of medicalizing variants of normal. Pediatrics 2013;131(5):991-2.

17. Orenstein SR, Hassall E, Furmaga-Jablonska W, Atkinson S, Raanan M. Multicenter, double-blind, randomized, placebo-controlled trial assessing efficacy \& safety of proton pump inhibitor lansoprazole in infants with symptoms of gastroesophageal reflux disease. J Pediatr 2009; 154(4):514-20.e4.

18. Hassall E. Over-prescription of acid-suppressing medications in infants: how it came about, why it's wrong, and what to do about it. J Pediatr 2012;160(2):193-8.

19. Melgar S, Nieuwdorp M. Are proton pump inhibitors affecting intestinal microbiota health? Gastroenterology 2015;149(4):848-50.

20. Douglas PS. Excessive crying and gastro-oesophageal reflux disease in infants: Misalignment of biology and culture. Med Hypotheses 2005;64(5):887-98.

21. Douglas P, Hill P. Managing infants who cry excessively in the first few months of life. BMJ 2011;343:d7772.

22. Orenstein S, MacGowan JD. Efficacy of conservative therapy as taught in the primary care setting for symptoms suggesting infant gastroesophageal reflux. J Pediatr 2008;152(3):310-4.

23. Davies HW, Satter E, Berlin KS, Sato AF, et al.
Reconceptualizing feeding and feeding disorders in interpersonal context: the case for a relational disorder. $J$ Fam Psychol 2006;20(3):409-17

24. Miller-Loncar C, Bigsby R, High P, Wallach M, Lester B. Infant colic and feeding difficulties. Arch Dis Child 2004;89(10):908-12.

25. Miranda BH, Millroy CJ. A quick snip-a study of the impact of outpatient tongue tie release on neonatal growth and breastfeeding. J Plast Reconstr Aesthet Surg 2010;63(9): e683-5.

26. DeSantis A, Coster W, Bigsby R, Lester B. Colic and fussing in infancy, and sensory processing at 3 to 8 years of age. Infant Ment Health J 2004;25(6):522-39.

27. Brodribb W, Fallon A, Jackson C, Hegney D. Breastfeeding and Australian GP registrars - their knowledge and attitudes. J Hum Lact 2008;24(4):422-30.

28. Douglas PS, Mares RE, Hill PS. Interdisciplinary perspectives on the management of the unsettled baby: Key strategies for improved outcomes. Aust J Prim Health 2012;18(4):332-8.

29. Smillie CM, Campbell SH, Iwinski S. Hyperlactation: how "left brained" rules for breastfeeding can wreak havoc with a natural process. Newborn Infant Nurs Rev 2005;5(1):49-58.

30. Huhtala V, Lehtonen L, Uvnas-Moberg K, Korvenranta H. Low plasma cholecystokinin levels in colicky infants. J Pediatr Gastroenterol Nutr 2003;37(1):42-6.

31. Evans K, Evans R, Simmer K. Effect of the method of breast feeding on breast engorgement, mastitis and infantile colic. Acta Paediatr 1995;84(8):849-52.

32. Sicherer SH. Epidemiology of food allergy. J Allergy Clin Immunol 2011;127(3):594-602.

33. Rona RJ, Keil T, Summers C, Gislason D, etal. The prevalence of food allergy: a meta-analysis. J Allergy Clin Immunol 2007;120(3):638-46.

34. Lothe L, Lindberg T, Jakobsson I. Cow's milk formula as a cause of infantile colic: a double-blind study. Pediatrics 1982;70(1):7-10.

35. Iacono G, Carroccio A, Montalto G, Cavataio F, et al. Severe infantile colic and food intolerance: a long-term prospective study. J Pediatr Gastroenterol Nutr 1991;12(3):332-5.

36. Hill D, Hudson IL, Sheffield LJ, Shelton MJ, et al. A low allergen diet is a significant intervention in infantile colic: results of a community-based study. J Allergy Clin Immunol 1995;96(6 Pt 1):886-92.

37. Hill DJ, Cameron DJ, Francis DE, González-Andaya AM, Hosking CS. Challenge confirmation of late onset reactions to extensively hydrolyzed formulas in infants with multiple food protein intolerance. J Allergy Clin Immunol 1995;96(3):386-94.

38. Hill DJ, Heine RG, Cameron DJ, Francis DE, Bines JE. The natural history of intolerance to soy and extensively hydrolyzed formula in infants with multiple food protein intolerance. J Pediatr 1999;135(1):118-21.

39. Jakobsson I, Lothe L, Ley D, Borschel MW. Effectiveness of casein hydrolysate feedings in infants with colic. Acta Paediatr 2000;89(1):18-21.

40. Hill DJ, Roy N, Heine RG, Hosking CS, et al. Effect of a lowallergen maternal diet on colic among breastfed infants: a randomized, controlled trial. Pediatrics 2005;116(5):e709-15.

41. Venter C, Brown T, Shah N, Walsh J, Fox AT. Diagnosis and management of non-IgE-mediated cow's milk allergy in infancy - a UK primary care practical guide. Clin Transl Allergy 2013;3(1):23

42. Jakobsson I, Lindberg T. Cow's milk proteins cause infantile colic in breast-fed infants: a double-blind crossover study. Pediatrics 1983;71(2):268-71.

43. Evans et al Evans RW, Fergusson DM, Allardyce RA, et al. Maternal diet and infantile colic in breast-fed infants. 
Lancet 1981;1:1340-2.

44. Forsyth BW. Colic and the effect of changing formulas: a double-blind, multiple-crossover study. J Pediatr 1989;115:521-6.

45. DeBoissieu D, DupontC. Allergy to extensively hydrolyzed cow's milk proteins in infants:safety and duration of amino acid-based formula. J Pediatr 2002;141(2):271-3.

46. Vanderhoof JA, Murray ND, Kaufman SS, Mack DR, et al. Intolerance to protein hydrolysate infant formulas: an underrecognized cause of gastrointestinal symptoms in infants. J Pediatr 1997;131(5):741-4.

47. Isolauri E, Sutas Y, Makinen-Kiljunen S, Oja SS, et al. Efficacy and safety of hydrolyzed cow milk and amino acid-derived formulas in infants with cow milk allergy. J Pediatr 1995;127(4):550-7.

48. Niggemann B, Binder C, Dupont C, Hadji S, et al. Prospective, controlled, multicenter study on the effect of an amino-acid-based formula in infants with cow's milk allergy/intolerance and atopic dermatitis. Pediatr Allergy Immunol 2001;12(2):78-82.

49. National Collaborating Center for Women`s and Children Health. Urinary tract infection in children: diagnosis, treatment and long-term management. Londres: RCOG Press; 2007. (NICE Clinical Guidelines, 54) [Acceso: 29 de febrero de 2016] Disponibleen:https:/ /www.nice.org.uk/ guidance/cg54/evidence/full-guideline-196566877.

50. Freedman SB, Al-Harthy N, Thull-Freedman J. The crying infant: diagnostic testing and frequency of serious underlying disease. Pediatrics 2009;123(3):841-8.

51. Kearney P, Malone A, Hayes T, Cole M, Hyland M. A trial of lactase in the management of infant colic. J Hum Nutr Diet 1998;11(4):281-5.

52. Kanabar D, Randhawa M, Clayton P. Improvement of symptoms in infant colic following reduction of lactose load with lactase. J Hum Nutr Diet 2001;14(5):359-63.

53. Anderson P. Infant Colic May Be Early Migraine. Medscape Medical News. Jul 3 2014. [Acceso: 29 de febrero de 2016]. Disponible en: http://www.medscape.com/ viewarticle/827825

54. Gelfand AA, Goadsby PJ, Allen IE. The relationship between migraine and infant colic: a systematic review and metaanalysis. Cephalalgia 2015;35(1):63-72.

55. Sillanpää M, Saarinen M. Infantile colic associated with childhood migraine: A prospective cohortstudy. Cephalalgia 2015;35(14):1246-51.

56. Roberts DM, Ostapchuk M, O'Brien JG. Infantile colic. Am Fam Physician 2004;70(4):735-40.

57. Hall B, Chesters J, Robinson A. Infantile colic: a systematic review of medical and conventional therapies. J Paediatr Child Health 2012;48(2):128-37.

58. Lucassen PL, Assendelft WJ, Gubbels JW, van Eijk JT, et al.
Effectiveness of treatments for infantile colic: systematic review. BMJ 1998;316(7144):1563-9.

59. Perry R,Hunt K,ErnstE. Nutritional supplements and other complementary medicines for infantile colic: a systematic review. Pediatrics 2011;127(4):720-33.

60. Savino F, Cordisco L, Tarasco V, Palumeri E, et al. Lactobacillus reuteri DSM 17938 in infantile colic: a randomized, double-blind placebo-controlled trial. Pediatrics 2010;126(3):e526-33.

61. Arikan D, Alp H, GözümS, OrbakZ, Cifçi EK. Effectiveness of massage, sucrose solution, herbal tea or hydrolysed formula in the treatment of infantile colic. J Clin Nurs 2008;17(13):1754-61.

62. Dupont C, Rivero M, Grillon C, Belaroussi N, et al .Alphalactalbumin-enriched and probiotic-supplemented infant formula in infants with colic: growth and gastrointestinal tolerance. Eur J Clin Nutr 2010;64(7):765-7.

63. Çetinkaya B, Ba bakkalZ. The effectiveness of aromatherapy massage using lavender oil as a treatment for infantile colic. Int J Nurs Pract 2012;18(2):164-9.

64. Van Sleuwen B, Engelberts AC, Boere-Boonekamp MM, Kuis W, et al. Swaddling: a systematic review. Pediatrics 2007;120(4):e1097-106.

65. Posadzki P, Ernst E. Is spinal manipulation effective for paediatric conditions? An overview of systematic reviews. Focus Altern Complement Ther 2012;17(1):22-6.

66. Posadzki P, Ernst E. Spinal manipulation: an update of a systematic review of systematic reviews. N Z Med J 2011;124(1340):55-71.

67. Alcantara J, Alcantara JD, Alcantara J. The chiropractic care of infants with colic: asystematic review of the literature. Explore (NY) 2011;7(3):168-74.

68. LandgrenK,Kvorning N,HallströmI. Acupuncture reduces crying in infants withinfantilecolic: a randomised, controlled, blind clinical study. Acupunct Med 2010;28(4):174-9.

69. Reinthal M, AnderssonS, Gustafsson M,Plos K, et al.Effects of minimal acupuncture in children with infantile colic-a prospective, quasi-randomised single blind controlled trial. Acupunct Med 2008;26(3):171-82.

70. Cohen-Silver J, Ratnapalan S. Management of infantile colic: a review. Clin Pediatr (Phila) 2009;48(1):14-7.

71. Newnham CA, Milgrom J, Skouteris H. Effectiveness of a modified Mother-InfantTransaction Program on outcomes for preterm infants from 3 to 24 months of age. Infant Behav Dev 2009;32(1):17-26.

72. Salisbury A, High P, Twomey JE, Dickstein S, et al. A randomized control trial of integrated care for families managing infant colic. Infant Ment Health J2012;33(2):110-22.

73. Kaley F, Reid V, Flynn E. The psychology of infant colic: a review of current research. Infant Ment Health J 2011;32(5):526-41.

\section{CORRECCIÓN}

Los autores del artículo especial: "Día Mundial del Riñón 2016. Prevención del legado de la nefropatía, con enfoque en la niñez", publicado en Arch Argent Pediatr 2016;114(2):147-153 solicitan que se aclare que han cometido un error en la Tabla 2. Donde dice HN: hipertensión corresponde NH: nefropatía hereditaria. 


\section{Anexo}

\section{Otras opciones terapéuticas}

\section{Tratamientos farmacológicos}

Los anticolinérgicos, como la diciclomina y el cimetopium, que reducen la actividad del músculo liso, han sido los más estudiados. En una revisión sistemática, dos estudios investigaron la diciclomina y observaron una mejoría en los cólicos. Sin embargo, graves efectos adversos, que incluyen problemas respiratorios y convulsiones, provocaron su restricción en niños de menos de 6 meses de edad en Norteamérica. ${ }^{56}$

Un estudio con cimetopium informó una mejoría significativa de los cólicos, pero se observó somnolencia. ${ }^{57}$ Ninguna de estas drogas ha sido autorizada en el Reino Unido.

La simeticona, que reduce la presencia de aire en la luz intestinal y es fácil de adquirir sin prescripción médica, ha sido estudiada en dos ensayos clínicos controlados y aleatorizados, y no se encontró diferencia respecto del placebo. ${ }^{56,58}$

\section{Medicinas complementarias y alternativas}

En ausencia de intervenciones farmacológicas seguras y efectivas, las terapias complementarias han adquirido un rol más preponderante en el manejo del lactante con cólicos. Dichas terapias abarcan desde terapias convencionales, como suplementos dietarios, soluciones azucaradas, extractos de hierbas o masajes, hasta opciones controversiales, como la quiropraxia.

\section{Suplementos a base de hierbas}

Una revisión sistemática identificó varios estudios sobre hierbas medicinales, tales como el extracto de hinojo y una mezcla de té de hierbas. Ambas infusiones mostraron una reducción en los cólicos del lactante. Sin embargo, la presencia de efectos adversos graves, como vómitos, somnolencia, constipación y pérdida del apetito, fue asociada a su ingesta. ${ }^{99,60}$

\section{Soluciones azucaradas}

Dos estudios compararon soluciones glucosadas con placebo y encontraron una mejoría de los cólicos. ${ }^{59,61}$ Sin embargo, existen reservas respecto de su impacto nutricional, en particular, el contenido de azúcar y alcohol, la falta de estandarización de sus formulaciones y la baja calidad de la evidencia científica.

\section{Probióticos}

Sustentado en la hipótesis de una flora intestinal aberrante que afecta la función intestinal y la producción de gas, que pueden contribuir a los síntomas, el uso de probióticos en los cólicos del lactante se ha tornado más común. Varios estudios han sido identificados en una revisión sistemática. ${ }^{59,60,62}$

Un ensayo clínico doble ciego, controlado con placebo, que incluyó la participación de 46 lactantes con cólicos, empleó una suspensión de Lactobacillus reuteri congelado y desecado. Se observó una respuesta significativa (50\% de reducción en el tiempo de llanto respecto de la línea de partida) en el grupo $L$. reuteri en los días 7 ( 20 vs. $8, P=0,006), 14$ ( 24 vs. 13 , $\mathrm{P}=0,007)$ y 21 ( 24 vs. $15, \mathrm{P}=0,036) \cdot{ }^{60}$ Otro estudio posterior, controlado y aleatorizado, informó buena ganancia de peso y tolerancia digestiva. ${ }^{62}$

\section{Masajes}

El efecto positivo del masaje en el que se usan aceites de aromaterapia se observó en un estudio. Sin embargo, no fue posible discriminar los efectos atribuibles al masaje o a los aceites. $.99,61,63$ Si bien otros estudios extraídos de una revisión sistemática mostraron cierta mejoría de los cólicos, en general, su calidad metodológica no fue satisfactoria.

\section{Arropado del bebé}

La envoltura o arropado del bebé ha sido utilizado tradicionalmente por algunos padres para calmar al bebé que llora. Una revisión sistemática sobre el tema observó que envolver al bebé redujo los síntomas de llanto comparado con el masaje en lactantes con llanto excesivo y daño cerebral. ${ }^{64}$ Sin embargo, existe un riesgo conocido de displasia de cadera, sobrecalentamiento y síndrome de muerte súbita, particularmente, si se coloca al bebé en posición prona. La base de la evidencia actual no sustenta el uso del arropado en el manejo del cólico del lactante.

\section{Tratamiento quiropráctico}

Como una terapia complementaria más controversial, la quiropraxia es, ocasionalmente, promovida como tratamiento para el bebé con llanto inconsolable. Esta práctica puede incluir 
osteopatía craneal y terapia de manipulación de la columna vertebral, pero no se limita a ellas.

La evaluación de las diferentes opciones terapéuticas en este campo representa un desafío, debido a la ausencia de ensayos clínicos controlados y aleatorizados de buena calidad. Más aun, efectos adversos, como la disección de la arteria vertebral, han sido informados. ${ }^{65,66}$ Se supone que la quiropraxia puede ejercer un efecto positivo sobre los síntomas; sin embargo, la literatura ha resaltado que esta respuesta puede ser más el resultado de una mejor capacidad de los padres para adaptarse a la situación que de una verdadera efectividad de esta práctica inusual. ${ }^{67}$ Por lo tanto, en la actualidad, la evidencia proveniente de la literatura es insuficiente como para recomendar el uso de la quiropraxia en bebés con cólicos.

\section{Acupuntura}

Varios ensayos controlados y aleatorizados han evaluado el efecto de la acupuntura, de los cuales solo dos de ellos han demostrado menor duración e intensidad en lactantes con cólicos. ${ }^{68,69}$

\section{Modificación del comportamiento}

Varias intervenciones sobre la conducta han sido identificadas. Orientadas a proveer tranquilidad y alivio a los padres de estos niños, ofrecen, a la vez, métodos alternativos para manejar esta situación. ${ }^{70}$
Una revisión sistemática identificó dos ensayos controlados, en los que una modificación de la interacción entre los padres y el bebé condujo a una significativa reducción de los cólicos, además de un beneficio adicional, como el avance más precoz en el desarrollo del bebé ${ }^{57,71}$ Esto ha sido atribuido a un incremento de la respuesta materna y al tiempo dedicado al bebé, que resultan en un aumento del nivel de alerta del niño.

En otro estudio, el compromiso de toda la familia al emplear un modelo de atención integrado se asoció con un alivio de los cólicos más evidente que el observado con los cuidados estándar. ${ }^{72}$

El empleo fortuito de la música ha demostrado reducir los síntomas en otro estudio. ${ }^{57}$

Se ha observado que es imperativo identificar estrategias efectivas para enfrentar las crisis de llanto continuo o intermitente, así como también los métodos para consolar al bebé, en la difícil tarea de asistir a los padres en una situación de estrés elevado, como lo es la de un bebé que llora inconsolablemente. ${ }^{73}$

Una revisión sistemática identificó dos estudios que abordan este aspecto: un estudio empleó atención de enfermería en el hogar, y el otro, asesoramiento en técnicas específicas para el manejo del bebé y la estimulación a través del paseo en auto en lactantes de 6 semanas de edad, lo que permitió una significativa reducción del estrés y la ansiedad de los padres. ${ }^{57,70}$ 